\title{
Limit theorems for probabilities of large deviations of a Galton-Watson process
}

\author{
S. V. NAGAEV and V. I. VAKHTEL
}

\begin{abstract}
We prove local and integral limit theorems for large deviations of Cramer type for a critical Galton-Watson branching process under the assumption that the radius of convergence of the generating function of the progeny is strictly greater than one. The proof is based on a modified Cramer approach which consists of construction of an auxiliary non-homogeneous in time branching process.

This research was supported by the Russian Foundation for Basic Research, grant 02-01-01252, and by INTAS, grants 99-01317, 00-265.
\end{abstract}

\section{INTRODUCTION}

Let $Z_{n}$ stand for a Galton-Watson process beginning with a single particle of zero generation. We set

$$
\begin{aligned}
p_{k} & =\mathbf{P}\left(Z_{1}=k\right), \quad k=0,1, \ldots, \\
f(s) & =\sum_{k=0}^{\infty} p_{k} s^{k},
\end{aligned}
$$

thus, $f(s)$ is the generating function of the progeny of an individual. Let $f_{k}(s)$ denote the $k$ th iteration of the function $f(s)$. We set $B=f^{\prime \prime}(1), C=f^{\prime \prime \prime}(1)$. Let $R$ stand for the convergence radius of the function $f(s)$.

For brevity, we set

$$
P_{n}(u)=\mathbf{P}\left(\frac{2 Z_{n}}{B n}>u \mid Z_{n}>0\right) .
$$

It is well known (see, e.g. [1], p. 39) that if $0<B<\infty$, then for any fixed $u$

$$
\lim _{n \rightarrow \infty} P_{n}(u)=e^{-u} .
$$

The first estimate of convergencerate in (1) was obtained in [2] under the condition $C<\infty$, namely,

$$
\Delta_{n}=\sup _{u}\left|P_{n}(u)-e^{-u}\right|=O\left(\frac{\ln ^{2} n}{n}\right) .
$$


We observe that the constants in this estimate have a more complicated relationship to the distribution of $Z_{1}$ as compared to the classical Berry-Esseen estimate.

If we set $u_{n}=\ln n-(2+\varepsilon) \ln \ln n$, where $\varepsilon>0$, then by (2)

$$
\lim _{n \rightarrow \infty} \sup _{u \leq u_{n}} e^{u} P_{n}(u)=1 .
$$

If $u>\ln n-(2-\varepsilon) \ln \ln n$, then (2) yields nothing more than the upper estimate

$$
P_{n}(u)=O\left(\frac{\ln ^{2} n}{n}\right)
$$

which does not depend on $u$. Thus, estimate (2) for large $u$ contains not so much information about the magnitude of the ratio $P_{n}(u) / e^{-u}$. As in the scheme of summation of independent random variables, the problem to estimate $P_{n}(u)$ for large $u$ is much easier to solve under the condition $R>1$.

Under this assumption, in [3] the inequality

$$
\mathbf{P}\left(Z_{n} \geq k\right)<\left(1+y_{0}\right)\left(1+\frac{1}{1 / y_{0}+B_{0} n / 2}\right)^{-k},
$$

is obtained, where $y_{0}$ is an arbitrary number from 0 to $R-1, B_{0}=f^{\prime \prime}\left(1+y_{0}\right)$.

It is easy to see that $1 / y_{0}+B_{0} n / 2$ attains its minimum if $y_{0}$ solves the equation $f^{\prime \prime \prime}(1+y) y^{2}=2 / n$, that is, $y_{0}=O(1 / \sqrt{n})$. Hence,

$$
\min _{y_{0}}\left(1 / y_{0}+B_{0} n / 2\right)=\frac{B n}{2}+O(\sqrt{n}) .
$$

Thus,

$$
\mathbf{P}\left(Z_{n} \geq k\right)<\left(1+\varepsilon_{n}\right) \exp \left(-\frac{2 k}{B n}\left(1-\eta_{n}\right)\right),
$$

where $\varepsilon_{n}>0, \eta_{n}>0$, and $\varepsilon_{n}=O(1 / \sqrt{n}), \eta_{n}=O(1 / \sqrt{n})$.

Inequality (4) is close to the Bernstein and Petrov inequalities (see, e.g. [4], Chapter 3, Section 5).

On the other hand, from (3) it follows that

$$
\mathbf{P}\left(Z_{n} \geq k\right)<\frac{2\left(1+\varepsilon_{n}\right)}{B n} \exp \left(-\frac{2 k}{B n}\right), \quad \varepsilon_{n} \rightarrow 0,
$$

for $k<B n(\ln n-(2+\varepsilon) \ln \ln n) / 2$, because [2]

$$
Q_{n}=\mathbf{P}\left(Z_{n}>0\right)=\frac{2}{B n}+\left(\frac{4 C}{3 B^{2}}-\frac{2}{B}\right) \frac{\ln n}{n^{2}}+o\left(\frac{\ln n}{n^{2}}\right) .
$$

We see that (5) is of less accuracy than (6) in the domain where the latter is valid, because (5) lacks the factor $2 /(B n)$.

Under the same assumption $R>1$, in [5] it is shown that (3) remains true if

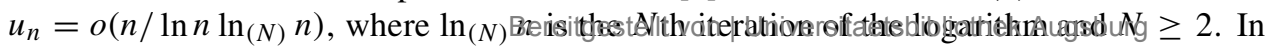


that paper, a local limit theorem is also proved for those $k$ which correspond to the domain $0<u<u_{n}$ under the additional assumption that $\operatorname{gcd}\left\{k: p_{k}>0\right\}=1$. More exactly,

$$
\mathbf{P}\left(Z_{n}=k\right)=\frac{4}{B^{2} n^{2}} \exp \left(-\frac{2 k}{B n}\right)(1+o(1))
$$

uniformly in $k=o\left(n^{2} / \ln n \ln _{(N)} n\right)$.

Starting from the analogy to the scheme of summation of independentrandom variables, we hypothesise that for $R>1$ there exists a domain of values of $u$ where

$$
P_{n}(u)=e^{-u} \Omega_{n}(u)(1+o(1))
$$

here $\Omega_{n}(u)$ is an explicitly calculated correction factor, that is, an analogue of the wellknown Cramer theorem is true [4]. The results obtained in the present paper are evidence in favour of this hypothesis.

Theorem 1. Let $R>1, k / n \rightarrow \infty, k=o\left(n^{2}\right)$. Then as $n \rightarrow \infty$

$$
\mathbf{P}\left(Z_{n}=k\right)=\frac{4 d}{B^{2} n^{2}} \exp \left\{-\frac{2 k}{B n}-\frac{2}{B} \gamma \frac{k}{n^{2}} \ln \left(\frac{k}{n}\right)\right\}\left(1+O\left(\frac{k}{n^{2}}+\frac{\ln n}{n}\right)\right),
$$

for $k$ divisible by $d=\operatorname{gcd}\left\{k: p_{k}>0\right\}$, where $\gamma=1-2 C /\left(3 B^{2}\right)$.

On the base of the local limit theorem, we arrive at the integral theorem on large deviations.

Theorem 2. Under the hypotheses of Theorem 1,

$$
\mathbf{P}\left(Z_{n} \geq k\right)=\frac{2}{B n} \exp \left\{-\frac{2 k}{B n}-\frac{2}{B} \gamma \frac{k}{n^{2}} \ln \left(\frac{k}{n}\right)\right\}\left(1+O\left(\frac{k}{n^{2}}+\frac{\ln ^{2} n}{n}\right)\right) .
$$

From this theorem we derive the exact boundaries where convergence to the exponential law takes place.

Corollary 1. If $u_{n}=o(n / \ln n)$, then relation (3) is true.

It is not difficult to see that these boundaries cannot be enlarged without additional constraints imposed on the process $Z_{n}$. If we set, say, $u_{n}=n / \ln n$, then by (11) and (7)

$$
\lim _{n \rightarrow \infty} e^{u_{n}} P_{n}\left(u_{n}\right)=\exp \left(-\frac{2 \gamma}{B}\right) \neq 1
$$

for $\gamma \neq 0$. In the case of $\gamma=0$, convergence to the exponential law takes place for all $u=o(n)$.

From (7) and (11) it follows that the equality

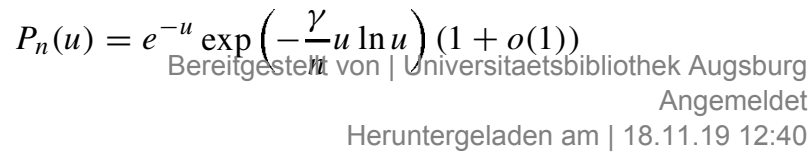


is true for $u=o(n)$, that is, $\Omega_{n}(u)$ in (9) satisfies the equality

$$
\Omega_{n}(u)=\exp \left(-\frac{\gamma}{n} u \ln u\right)
$$

We observe that, in contrast to the classical Cramer theorem, $\ln \Omega_{n}(u)$ depends on the second and third moments of the initial distribution only. This is likely due to the fact that the domain where the correction factor is of importance is quite narrow.

Our way to derive relations (10) and (11) differs much from that used in [5]. The proof of Theorem 1 is based on a modified Cramer method (see, e.g. [4], Chapter 8, Section 2), which consists of the following.

Let $X$ be a random variable that takes non-negativeinteger values only. We assume that the radius of convergence of the function $\rho(s)=\mathbf{E} s^{X}$ is strictly greater that one. Then for any $r$ such that $\rho(r)<\infty$ we set $\rho_{r}(s)=\rho(r s) / \rho(r)$.

The random variable $X(r)$ such that $\mathbf{E} s^{X(r)}=\rho_{r}(s)$ is referred to as the Cramer transform of the random variable $X$. With the use of Cramer transforms of the random variable $Z_{1}$, we construct a non-homogeneous in time Galton-Watson process $Y_{k}, k=1, \ldots, n$, such that the distribution of the progeny of an individualin the $(k-1)$ th generation is defined by the generating function $f\left(r_{k-1} s\right) / f\left(r_{k-1}\right)$, where the parameters $r_{k}$ of the Cramer transform are calculated by the recurrence relation $r_{k}=f\left(r_{k-1}\right)$. Then the distributions of the initial process and the auxiliary one are related as follows:

$$
\mathbf{P}\left(Z_{n}=k\right)=f_{n}\left(r_{0}\right) r_{0}^{-k} \mathbf{P}\left(Y_{n}=k\right), \quad \mathbf{P}\left(Z_{n} \geq k\right)=f_{n}\left(r_{0}\right) \mathbf{E}\left\{r_{0}^{-Y_{n}} ; Y_{n} \geq k\right\} .
$$

The parameter $r_{0}$ is chosen so that the large deviations of the initial process become normal ones for the auxiliary process.

Non-homogeneous in time branching processes are studied in [6], where conditions of convergence to the exponential law are obtained, as well as an estimate of the convergence rate, which coincides with (2) for a critical Galton-Watson process.

In the classical Cramer theorem, the asymptotic behaviour of the mathematical expectation which connects the distributions of the initial and auxiliary sums is found with the use of the Berry-Esseen inequality. In the case of branching processes, this method does not allow us to find the asymptotic formula for $\mathbf{E}\left\{r_{0}^{-Y_{n}} ; Y_{n} \geq k\right\}$ because the Berry-Esseen type estimate for the auxiliary process becomes too rough.

If $d>1$, we may reduce the case to aperiodic one. We consider the process $Z_{n}^{*}$ constructed by the generating function

$$
g(s)=\left(f\left(s^{1 / d}\right)\right)^{d}=\sum_{i=0}^{\infty} p_{i}^{*} s^{i} .
$$

It is obvious that the convergence radius of $g(s)$ is also greater than one and

$$
d^{*}=\operatorname{gcd}\left\{k: p_{k}^{*}>0\right\}=1 .
$$

Besides, $B^{*}=g^{\prime \prime}(1)=B / d, C^{*}=g^{\prime \prime \prime}(1)=C / d^{2}$. Therefore, $\gamma^{*}=\gamma$.

It is not difficult to see that for any $n \geq 1$ 
This equality means that the process $Z_{n}^{*}$ admits the representation

$$
Z_{n}^{*}=\frac{1}{d}\left(Z_{n}^{(1)}+\ldots+Z_{n}^{(d)}\right)
$$

where $Z_{n}^{(i)}, i=1, \ldots, d$, are independent random variables distributed as $Z_{n}$. This representation yields the inequality

$$
\mathbf{P}\left(Z_{n}^{*}=k\right)=\mathbf{P}\left(Z_{n}^{(1)}+\ldots+Z_{n}^{(d)}=k d\right) \geq d\left(1-Q_{n}\right)^{d-1} \mathbf{P}\left(Z_{n}=k d\right) .
$$

On the other hand [7],

$$
\mathbf{P}\left(Z_{n}=k d\right) \geq \mathbf{P}\left(Z_{n-1}^{*}=k\right) \sum_{l=1}^{\infty} l p_{l d} \mathbf{P}^{l-1}\left(Z_{n}^{*}=0\right) .
$$

It is not difficult to see that

$$
\sum_{l=1}^{\infty} l p_{l d} \mathbf{P}^{l-1}\left(Z_{n}^{*}=0\right)=\left.\left(f\left(s^{1 / d}\right)\right)^{\prime}\right|_{s=1}+O\left(\mathbf{P}\left(Z_{n}^{*}>0\right)\right)=\frac{1}{d}+O\left(n^{-1}\right) .
$$

From (14), (15), and the last relation it follows that

$$
\begin{aligned}
& \mathbf{P}\left(Z_{n}=k d\right) \leq \frac{1}{d} \mathbf{P}\left(Z_{n}^{*}=k\right)\left(1+O\left(n^{-1}\right)\right), \\
& \mathbf{P}\left(Z_{n}=k d\right) \geq \frac{1}{d} \mathbf{P}\left(Z_{n-1}^{*}=k\right)\left(1+O\left(n^{-1}\right)\right)
\end{aligned}
$$

uniformly in all $k$.

If we assume that the theorem is true for the aperiodic case, then, applying it to the process $Z_{n}^{*}$, we obtain

$$
\mathbf{P}\left(Z_{n}^{*}=k\right)=\frac{4 d^{2}}{B^{2} n^{2}} \exp \left\{-\frac{2 k d}{B n}-\frac{2}{B} \gamma \frac{k d}{n^{2}} \ln \left(\frac{k}{n}\right)\right\}\left(1+O\left(\frac{k}{n^{2}}+\frac{\ln n}{n}\right)\right) .
$$

Hence it follows that

$$
\mathbf{P}\left(Z_{n-1}^{*}=k\right)=\mathbf{P}\left(Z_{n}^{*}=k\right)\left(1+O\left(\frac{k}{n^{2}}+\frac{\ln n}{n}\right)\right)
$$

for $k=o\left(n^{2}\right)$. The two last relations and inequalities (16), (17) imply the equality

$$
\mathbf{P}\left(Z_{n}=k d\right)=\frac{4 d}{B^{2} n^{2}} \exp \left\{-\frac{2 k d}{B n}-\frac{2}{B} \gamma \frac{k d}{n^{2}} \ln \left(\frac{k}{n}\right)\right\}\left(1+O\left(\frac{k}{n^{2}}+\frac{\ln n}{n}\right)\right),
$$

that is, it is proved that validity of (10) in the aperiodic case implies validity of (10) for an arbitrary $f(s)$. Similarly it is proved that (11) remains true for $d>1$ as well. Thus, it suffices to prove Theorems 1 and 2 under the condition $d=1$. It is necessary to note that such a reduction to the aperiodic case was used in [7] while proving a local limit theorem. But they derived a similar to (14) estimate without use of representation (13), which contributed to the difficulties they met. 


\section{AUXILIARY RESULTS}

Lemma 1. Let $0<y_{0}<R-1$, and let the sequence $y_{j}$ be defined by the equation

$$
y_{j+1}=f^{-1}\left(1+y_{j}\right)-1 \text {. }
$$

Then

$$
\begin{aligned}
y_{j}=\frac{y_{0}}{1+B j y_{0} / 2} & +\left(\frac{B}{2}-\frac{C}{3 B}\right) \frac{y_{0}^{2}}{\left(1+B j y_{0} / 2\right)^{2}} \ln \left(1+\frac{B j y_{0}}{2}\right) \\
& +O\left(\frac{y_{0}^{2}}{\left(1+B j y_{0} / 2\right)^{2}}\right) .
\end{aligned}
$$

This lemma, as well as its proof, is very similar to Theorem 3 in [2].

Proof. From the definition of $y_{j}$ it follows that

$$
y_{j}=f\left(1+y_{j+1}\right)-1 \text {. }
$$

It is not difficult to see that $f(1+y) \geq 1+y$ for $y \geq 0$. Therefore, the sequence $y_{j}$ decreases. Hence the existence of a limit of $y_{j}$ as $j \rightarrow \infty$ follows. It is obvious that this limit has to satisfy the equation $y=f(1+y)-1$. But the only root of this equation is $y=0$. This means that $y_{j} \rightarrow 0$ as $j \rightarrow \infty$ and $\sum_{i=0}^{j} y_{i}=o(j)$.

Expanding $f(1+z)$ into the Taylor series, we obtain

$$
y_{j}=y_{j+1}+\frac{B}{2} y_{j+1}^{2}+\frac{C}{6} y_{j+1}^{3}+O\left(y_{j+1}^{4}\right) .
$$

From this relation it follows that

$$
\begin{aligned}
\frac{y_{j+1}}{y_{j}} & =\frac{1}{1+B y_{j+1} / 2+O\left(y_{j+1}^{2}\right)}=1-\frac{B y_{j+1}}{2}+O\left(y_{j+1}^{2}\right) \\
& =1-\frac{B y_{j}}{2}+O\left(y_{j}^{2}\right) .
\end{aligned}
$$

Dividing both parts of (19) by $y_{j} y_{j+1}$ and making use of (20) we obtain

$$
\begin{aligned}
\frac{1}{y_{j+1}} & =\frac{1}{y_{j}}+\frac{B}{2}-\left(\frac{B^{2}}{4}-\frac{C}{6}\right) y_{j}+O\left(y_{j}^{2}\right)=\ldots \\
& =\frac{1}{y_{0}}+\frac{B j}{2}-\left(\frac{B^{2}}{4}-\frac{C}{6}\right) \sum_{i=0}^{j} y_{i}+O\left(\sum_{i=0}^{j} y_{i}^{2}\right) .
\end{aligned}
$$

Therefore,

$$
\frac{1}{y_{j}}=\frac{1}{\text { Berejdgestedt }}+\frac{B}{\text { von }}+o(j \text { iniviersitaetsbibliothek Augsburg }
$$


This, in its turn, implies that

$$
y_{j}=\frac{y_{0}}{1+B j y_{0} / 2}(1+o(1))
$$

and

$$
\begin{aligned}
\sum_{i=0}^{j-1} y_{i}^{2} & =O\left(\sum_{i=0}^{j-1} \frac{y_{0}^{2}}{\left(1+B i y_{0} / 2\right)^{2}}\right) \\
& =O\left(y_{0} \int_{0}^{\infty} \frac{y_{0} d x}{\left(1+B x y_{0} / 2\right)^{2}}\right)=O\left(y_{0}\right) .
\end{aligned}
$$

Now we study the behaviour of $\sum_{i=0}^{j-1} y_{i}$. With the use of the inequalities

$$
\begin{aligned}
& \sum_{i=0}^{j-1} \frac{y_{0}}{1+B i y_{0} / 2} \leq y_{0}+\int_{0}^{j} \frac{y_{0} d x}{1+B x y_{0} / 2}=y_{0}+\frac{2}{B} \ln \left(1+B j y_{0} / 2\right), \\
& \sum_{i=0}^{j-1} \frac{y_{0}}{1+B i y_{0} / 2} \geq \int_{0}^{j} \frac{y_{0} d x}{1+B x y_{0} / 2}=\frac{2}{B} \ln \left(1+B j y_{0} / 2\right),
\end{aligned}
$$

we arrive at the relation

$$
\sum_{i=0}^{j-1} y_{i}=\sum_{i=0}^{j-1} \frac{y_{0}}{1+B i y_{0} / 2}(1+o(1))=O\left(\ln \left(1+B j y_{0} / 2\right)\right) .
$$

From (21) and the last equality we obtain

$$
\frac{1}{y_{j}}=\frac{1}{y_{0}}+\frac{B}{2} j+O\left(\ln \left(1+B j y_{0} / 2\right)\right) .
$$

Hence it follows that

$$
y_{j}=\frac{y_{0}}{1+B j y_{0} / 2}+O\left(\frac{y_{0}^{2}}{\left(1+B j y_{0} / 2\right)^{2}} \ln \left(1+B j y_{0} / 2\right)\right) .
$$

With the use of this relation we estimate $\sum_{i=0}^{j-1} y_{i}$ with the accuracy required to prove the lemma:

$$
\sum_{i=0}^{j-1} y_{i}=\sum_{i=0}^{j-1} \frac{y_{0}}{1+B i y_{0} / 2}+O\left(y_{0}^{2} \sum_{i=0}^{j-1} \frac{\ln \left(1+B i y_{0} / 2\right)}{\left(1+B i y_{0} / 2\right)^{2}}\right) .
$$

It is not difficult to see that the second term in the right-hand side of the preceding equality is $O\left(y_{0}\right)$.

Making use of (24) and (25) again, we obtain

$$
\begin{array}{r}
\sum_{i=0}^{j-1} y_{i}=\frac{2}{B} \ln \left(1+B j y_{0} / 2\right)+O\left(y_{0}\right) . \\
\text { Bereitgestellt von | Universitaetsbibliothek Augsburg } \\
\text { Angemeldet } \\
\text { Heruntergeladen am | 18.11.19 12:40 }
\end{array}
$$


Using (21), (23), and (26), we arrive at

$$
\frac{1}{y_{j}}=\frac{1}{y_{0}}+\frac{B}{2} j-\left(\frac{B}{2}-\frac{C}{3 B}\right) \ln \left(1+B j y_{0} / 2\right)+O\left(y_{0}\right) .
$$

The lemma is thus proved.

We set $r_{0}=1+y_{n}$ and for $i=1, \ldots, n$ define the probability generating function

$$
g_{j}(s)=\frac{f\left(s f_{j-1}\left(r_{0}\right)\right)}{f_{j}\left(r_{0}\right)} .
$$

We introduce

$$
A_{j}=g_{j}^{\prime}(1), \quad A(j)=A_{1} \ldots A_{j}, \quad B_{j}=g_{j}^{\prime \prime}(1), \quad T(l)=\sum_{j=0}^{l-1} \frac{B_{j+1}}{2 A_{j+1}} A(j) .
$$

Lemma 2. If $y_{0} \rightarrow 0$, then

$$
\begin{aligned}
& A(j)=\left(\frac{1+B n y_{0} / 2}{1+B(n-j) y_{0} / 2}\right)^{2}\left(1+O\left(y_{0}\right)\right), \\
& T(l)=\frac{B l\left(1+B n y_{0} / 2\right)}{2\left(1+B(n-l) y_{0} / 2\right)}\left(1+O\left(y_{0}\right)\right)
\end{aligned}
$$

uniformly in $1 \leq j \leq n$ and $1 \leq l \leq n$.

Proof. From the definitions of $A(j)$ and $g_{j}(s)$ it immediately follows that

$$
A(j)=\frac{r_{0}}{f_{j}\left(r_{0}\right)} \prod_{i=0}^{j-1} f^{\prime}\left(1+y_{n-i}\right)=\frac{r_{0}}{f_{j}\left(r_{0}\right)} \exp \left\{\sum_{i=0}^{j-1} \ln f^{\prime}\left(1+y_{n-i}\right)\right\} .
$$

With the use of the relations

$$
f^{\prime}(1+z)=1+B z+O\left(z^{2}\right), \quad \ln (1+t)=t+O\left(t^{2}\right),
$$

we obtain

$$
A(j)=\frac{r_{0}}{f_{j}\left(r_{0}\right)} \exp \left\{B \sum_{i=0}^{j-1} y_{n-i}+O\left(\sum_{i=0}^{j-1} y_{n-i}^{2}\right)\right\}
$$

By virtue of (23) and (26),

$$
\begin{array}{r}
\left.A(j)=\frac{r_{0}}{f_{j}\left(r_{0}\right)} \exp \left\{2 \ln \left(1+B n y_{0}\right)-2 \ln \left(1+B(n-j) y_{0}\right)\right)+O\left(y_{0}\right)\right\} \\
=\frac{r_{0}}{f_{j}\left(r_{0}\right)}\left(\frac{1+B n y_{0} / 2}{1+B(n \text { Bejeltygestelt von | Universitaetsbibliothek Augsburg }}\right. \text { Angemeldet } \\
\text { Heruntergeladen am | 18.11.19 12:40 }
\end{array}
$$


It is not difficult to see that

$$
\frac{r_{0}}{f_{j}\left(r_{0}\right)}=\frac{1+y_{n}}{1+y_{n-j}}=1+O\left(y_{0}\right) .
$$

The two last relations imply (27).

Let us turn to the proof of (28). It is obvious that

$$
B_{i}=B\left(1+O\left(y_{0}\right)\right), \quad A_{i}=1+O\left(y_{0}\right)
$$

uniformly in $i$. Therefore,

$$
T(l)=\left(\frac{B}{2} \sum_{j=0}^{l-1} A(j)\right)\left(1+O\left(y_{0}\right)\right) .
$$

Using (27), we obtain

$$
T(l)=\sum_{j=0}^{l-1} \frac{B}{2}\left(\frac{1+B n y_{0} / 2}{1+B(n-j) y_{0} / 2}\right)^{2}\left(1+O\left(y_{0}\right)\right) .
$$

Furthermore, since the function $B /\left(2\left(1+B x y_{0} / 2\right)^{2}\right)$ decreases, the inequalities

$$
\int_{n-l+1}^{n+1} \frac{B d x}{2\left(1+B x y_{0} / 2\right)^{2}} \leq \sum_{j=0}^{l-1}\left(1+B(n-j) y_{0} / 2\right)^{-2} \leq \int_{n-l}^{n} \frac{B d x}{2\left(1+B x y_{0} / 2\right)^{2}}
$$

are true. It is not difficult to see that each integral in the preceding inequality is

$$
\frac{B l}{2\left(1+B n y_{0} / 2\right)\left(1+B(n-l) y_{0} / 2\right)}\left(1+O\left(y_{0}\right)\right) \text {. }
$$

Therefore,

$$
\sum_{j=0}^{l-1}\left(1+B(n-j) y_{0} / 2\right)^{-2}=\frac{B l}{2\left(1+B n y_{0} / 2\right)\left(1+B(n-l) y_{0} / 2\right)}\left(1+O\left(y_{0}\right)\right) .
$$

From (29) and (30) we arrive at the required relation.

Let

$$
\rho(s)=\sum_{k=0}^{\infty} \rho_{k} s^{k}
$$

we set

$$
\|\rho\|_{1}=\sum_{k=0}^{\infty}\left|\rho_{k}\right|
$$

It is obvious that $\|\cdot\|_{1}$ possesses all poneptentieslof can nomin.ersitaetsbibliothek Augsburg 
Lemma 3. Let $\rho(s)$ be a probability generating function. We set $a=\rho^{\prime}(1), b=\rho^{\prime \prime}(1)$. Then

$$
\frac{1}{1-\rho(s)}=\frac{1}{a(1-s)}+\frac{b}{2 a^{2}}+(\delta(s)-\eta(s)) \frac{(1-s)^{2}}{1-\rho(s)}
$$

where

$$
\delta(s)=\frac{b(\rho(s)-1-a(s-1))}{2 a^{2}(s-1)^{2}}, \quad \eta(s)=\frac{\rho(s)-1-a(s-1)-b(s-1)^{2} / 2}{a(s-1)^{3}} .
$$

If we introduce the extra constraint $c=\rho^{\prime \prime \prime}(1)<\infty$, then

$$
\|\delta(s)-\eta(s)\|_{1} \leq \frac{b^{2}}{4 a^{2}}+\frac{c}{6 a} .
$$

Proof. From the equality

$$
\frac{1}{1-\rho(s)}-\frac{1}{a(1-s)}=\left(\frac{\rho(s)-1-a(s-1)}{a(s-1)^{2}}\right) \frac{(1-s)}{1-\rho(s)}
$$

it follows that

$$
\frac{1}{1-\rho(s)}-\frac{1}{a(1-s)}=\frac{b(1-s)}{2 a(1-\rho(s))}-\eta(s) \frac{(1-s)^{2}}{1-\rho(s)} .
$$

Furthermore, (32) yields

$$
\frac{1-s}{1-\rho(s)}=\frac{1}{a}+\left(\frac{\rho(s)-1-a(s-1)}{a(s-1)^{2}}\right) \frac{(1-s)^{2}}{1-\rho(s)} .
$$

Substituting this into (33), we finally arrive at relation (31).

It is easy to verify that

$$
\frac{\rho(s)-1-a(s-1)}{(s-1)^{2}}=\sum_{k=2}^{\infty} \rho_{k} \sum_{i=1}^{k-1} \sum_{j=0}^{i-1} s^{j} .
$$

Therefore,

$$
\left\|\frac{\rho(s)-1-a(s-1)}{(s-1)^{2}}\right\|_{1}=\frac{b}{2} .
$$

Similarly we find that

$$
\left\|\frac{\rho(s)-1-a(s-1)-b(s-1)^{2}}{(s-1)^{3}}\right\|_{1}=\frac{c}{6} .
$$

Inequalities (35) and (36) yield the estimate

$$
\|\delta(s)-\eta(s)\|_{1} \leq\|\delta(s)\|_{1}+\|\eta(s)\|_{1}=\frac{b^{2}}{4 a^{2}}+\frac{c}{6 a},
$$

which proves the lemma. 
We set

$$
G_{i}(s)=g_{i} \circ \ldots \circ g_{1}(s)=f_{i}\left(r_{0} s\right) / f_{i}\left(r_{0}\right), \quad q_{i}(s)=1-G_{i}(s) .
$$

In what follows we assume that $y_{0} \rightarrow 0$.

Lemma 4. For any $i \geq 1$, the inequality

$$
\left\|q_{i}(s)\right\|_{1} \leq 2\left(Q_{i}+y_{n-i}\right)
$$

is true. From this bound it follows that $q_{i}(s) \rightarrow 0$ as $i \rightarrow \infty$ uniformly in $s$ inside the unit disk.

Proof. Let

$$
\rho(s)=\sum_{k=0}^{\infty} \rho_{k} s^{k}
$$

be a probability generating function. It is easy to check that

$$
\|1-\rho(s)\|_{1}=\left(1-\rho_{0}\right)+\sum_{k=1}^{\infty} \rho_{k}=2(1-\rho(0)) .
$$

Applying this equality to $G_{i}(s)$, we obtain

$$
\left\|q_{i}(s)\right\|_{1}=2\left(1-\frac{f_{i}(0)}{f_{i}\left(r_{0}\right)}\right)=2 \frac{f_{i}\left(r_{0}\right)-f_{i}(0)}{f_{i}\left(r_{0}\right)} \leq 2\left(Q_{i}+f_{i}\left(r_{0}\right)-1\right) .
$$

Since $\left.f_{(} r_{0}\right)=1+y_{n-i}$, we arrive at inequality (37).

The convergence of $\left\|q_{i}(s)\right\|_{1}$ to zero follows from the facts that $Q_{i} \rightarrow 0$ as $i \rightarrow \infty$ and $y_{0} \rightarrow 0$. The uniform inside the unit disk convergence of $q_{i}(s)$ to zero follows from the inequality $\sup _{|s| \leq 1}\left|q_{i}(s)\right| \leq\left\|q_{i}(s)\right\|_{1}$.

Lemma 5. Let $\operatorname{gcd}\left\{k: p_{k}>0\right\}=1$. Then there exists $N$ such that

$$
q_{j}(s)=\frac{A(j)}{(1-s)^{-1}+T(j)}\left(1-\frac{R_{N}(s)+\sum_{i=N}^{j-1} A(i) q_{i}(s) C_{i}(s)}{(1-s)^{-1}+T(j)}\right)^{-1}
$$

for $j \geq N,|s| \leq 1$, where $C_{i}(s), R_{N}(s)$ are some analytic in the unit disk functions, and $\left\|C_{i}(s)\right\|_{1} \leq C,\left\|R_{N}(s)\right\|_{1}<\infty$.

Proof. Substituting $\rho=g_{j+1}, s=G_{j}$ into (31), we obtain the equality

$$
\frac{1}{q_{j+1}(s)}=\frac{1}{A_{j+1} q_{j}(s)}+\frac{B_{j+1}}{2 A_{j+1}^{2}}-d_{j+1}(s) \frac{q_{j}^{2}(s)}{A_{j+1} q_{j+1}(s)},
$$

where

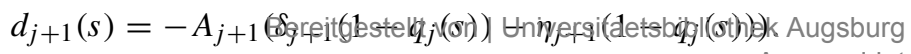


Multiplying both sides of this equality by $A(j+1)$, we arrive at the recurrence relation

$$
b_{j+1}(s)=b_{j}(s)+\frac{B_{j+1}}{2 A_{j+1}} A(j)-d_{j+1}(s) A(j) \frac{q_{j}^{2}(s)}{q_{j+1}(s)},
$$

where $b_{j}(s)=A(j) / q_{j}(s)$. From this equality we easily derive the following expansion of the function $b_{j}(s)$ :

$$
\begin{aligned}
b_{j}(s) & =b_{N}(s)+T(j)-T(N)-\sum_{i=N}^{j-1} A(i) q_{i}(s) C_{i}(s) \\
& =\frac{1}{1-s}+T(j)-R_{N}(s)-\sum_{i=N}^{j-1} A(i) q_{i}(s) C_{i}(s),
\end{aligned}
$$

where

$$
C_{i}(s)=d_{i+1}(s) q_{i} / q_{i+1}(s), \quad R_{N}(s)=(1-s)^{-1}+T(N)-b_{N}(s),
$$

$N$ is an arbitrary positive integer less than $j$. This proves representation (38).

It remains to prove that the functions $C_{i}(s), R_{N}(s)$ are analytic and their norms are bounded.

From Lemma 3 it follows that

$$
\left\|d_{j+1}\right\|_{1} \leq A_{j+1}\left(\left\|\delta_{j+1}\right\|_{1}+\left\|\eta_{j+1}\right\|_{1}\right) \leq\left(\frac{\left(g_{j+1}^{\prime \prime}(1)\right)^{2}}{4 A_{j+1}}+\frac{g_{j+1}^{\prime \prime \prime}(1)}{6}\right) .
$$

From this relation it follows that $\left\|d_{j+1}\right\|_{1}$ are uniformly bounded.

If we assume that $\operatorname{gcd}\left\{k: \rho_{k}>0\right\}=1$, then the function $(1-\rho(s)) /(1-s)$ has no zeros inside the unit disk. If $s_{0}$ were a zero of this function, then $\rho\left(s_{0}\right)=1$. Therefore, $\left|s_{0}\right|=1$ and $s_{0}^{k}=1$ for any $k$ such that $\rho_{k}>0$. From the aperiodicity condition it follows that the only point which satisfies these conditions is $s_{0}=1$. But this point does not make the function $(1-\rho(s)) /(1-s)$ vanish because

$$
\left.\frac{1-\rho(s)}{1-s}\right|_{s=1}=\left.\sum_{k=1}^{\infty} \rho_{k}\left(1+\ldots+s^{k-1}\right)\right|_{s=1}=a .
$$

By virtue of the Tauberian theorem due to Wiener (see, e.g. [8]), the function $(1-s) /(1-\rho(s))$ is analytic and $\|(1-s) /(1-\rho(s))\|_{1}<\infty$.

From aperiodicity of the sequence $p_{k}$ it follows that the coefficients of the function $g_{j}(s)$ also form an aperiodic sequence. Therefore,

$$
\left\|\frac{q_{j}}{q_{j+1}}\right\|_{1} \leq\left\|\frac{1-s}{1-g_{j+1}(s)}\right\|_{1}<\infty .
$$

Furthermore, relation (34) yields the estimate

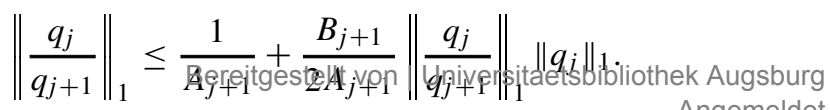


Making use of Lemma 4, we conclude that

$$
\left\|\frac{q_{j}}{q_{j+1}}\right\|_{1} \leq 2
$$

for all $j$ exceeding a certain threshold value and for sufficiently small $y_{0}$.

From (39) and (40) it follows that the norms of $C_{i}(s)$ are bounded for all $i$ no less than a certain $N$.

Let us consider the function $R_{N}(s)$. It is clear that

$$
\left\|R_{N}(s)\right\|_{1} \leq T(N)+A(N)\left\|\frac{1}{q_{N}(s)}-\frac{1}{A(N)(1-s)}\right\|_{1} .
$$

Setting $\rho(s)=G_{N}(s)$ in (32), we obtain

$$
\frac{1}{q_{N}(s)}-\frac{1}{A(N)(1-s)}=\left(\frac{G_{N}(s)-1-A(N)(s-1)}{A(N)(s-1)^{2}}\right) \frac{1-s}{q_{N}(s)} .
$$

Making use of equality (35), we find that

$$
\left\|\frac{G_{N}(s)-1-A(N)(s-1)}{A(N)(s-1)^{2}}\right\|_{1}=\frac{G_{N}^{\prime \prime}(1)}{2 A(N)}=\frac{T(N)}{2 A(N)} .
$$

Therefore,

$$
\left\|\frac{1}{q_{N}(s)}-\frac{1}{A(N)(1-s)}\right\|_{1} \leq \frac{T(N)}{2}\left\|\frac{1-s}{q_{N}(s)}\right\|_{1} .
$$

The boundedness of $\left\|(1-s) / q_{N}(s)\right\|_{1}$ follows from aperiodicity of the coefficients of the function $G_{N}(s)$ and the Wiener theorem mentioned above. Finally, we see that $R_{N}(s)$ is bounded in the norm.

Lemma 6. As $i \rightarrow \infty$,

$$
q_{i}(s)\left(\frac{(1-s)^{-1}+T(i)}{A(i)}\right) \rightarrow 1
$$

uniformly in all s inside the unit disk.

Proof. It is clear that in order to prove the lemma it suffices to check that the function

$$
\frac{R_{N}(s)+\sum_{i=N}^{j-1} A(i) q_{i}(s) C_{i}(s)}{(1-s)^{-1}+T(j)}
$$

converges to zero uniformly in the unit disk. Let $\alpha_{i}, \beta_{i}$ be numerical sequences satisfying the conditions

$$
\sum_{i=1}^{n} \alpha_{i} \rightarrow \infty, \quad \beta_{i} \rightarrow 0
$$

Then, as we easily see,

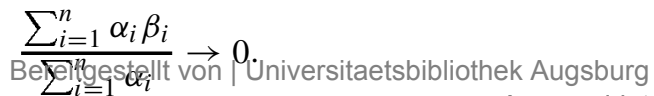


From the definition of $T(j)$ it follows that

$$
\sum_{i=N}^{j-1} A(i)=O(T(j))
$$

By virtue of Lemma $4, q_{i}(0) \rightarrow 0$. From the abovesaid it follows that

$$
\frac{\sum_{i=N}^{j-1} A(i) q_{i}(0)}{T(j)} \rightarrow 0
$$

It is obvious that

$$
\left\|\frac{R_{N}(s)+\sum_{i=N}^{j-1} A(i) q_{i}(s) C_{i}(s)}{(1-s)^{-1}+T(j)}\right\|_{1} \leq \frac{\left\|R_{N}(s)\right\|_{1}+\sum_{i=N}^{j-1} A(i)\left\|q_{i}(s)\right\|_{1}\left\|C_{i}(s)\right\|_{1}}{T(j)} .
$$

Since $\left\|R_{N}(s)\right\|_{1}<\infty$ and $T(j) \rightarrow \infty$, the relation $\left\|R_{N}(s)\right\|_{1} / T(j) \rightarrow 0$ is true. Making use of Lemmas 4 and 5, we obtain

$$
\frac{1}{T(j)} \sum_{i=N}^{j-1} A(i)\left\|q_{i}(s)\right\|_{1}\left\|C_{i}(s)\right\|_{1} \leq \frac{2 C}{T(j)} \sum_{i=N}^{j-1} A(i) q_{i}(0) .
$$

In view of (42), the right-hand side of the last inequality tends to zero. Thus,

$$
\left\|\frac{R_{N}(s)+\sum_{i=N}^{j-1} A(i) q_{i}(s) C_{i}(s)}{(1-s)^{-1}+T(j)}\right\|_{1} \rightarrow 0 .
$$

This, in turn, means that the function

$$
\frac{R_{N}(s)+\sum_{i=N}^{j-1} A(i) q_{i}(s) C_{i}(s)}{(1-s)^{-1}+T(j)}
$$

converges to zero uniformly in $s$ inside the unit disk. The lemma is thus proved.

Let $\rho(s)$ be a power series. Let $a_{k}[\rho(s)]$ denote the $k$ th coefficient of this series.

Lemma 7. Let $\rho(s)$ be a probability generating function and $\rho^{\prime}(1)<\infty$. Then the inequality

$$
a_{l}[\rho(s)] \leq \frac{1}{l} \int_{-a}^{a}\left|\rho^{\prime}\left(e^{i t}\right)\right| d t
$$

holds for any $a>0$.

Proof. It is obvious that the function $\rho^{\prime}(s) / \rho^{\prime}(1)$ is a probability generating function. The following bound for the concentration function is well known [4]:

$$
\begin{array}{r}
\left.\sup _{x} \mathbf{P}(X=x) \underset{\text { Bereitgestèlt von } \mid}{<}\right)^{-1} \int_{-a}^{a}|\varphi(t)| d t \text { erstaetsbibliothek Augsburg } \\
\text { Angemeldet } \\
\text { Heruntergeladen am | 18.11.19 12:40 }
\end{array}
$$


where $\varphi(t)$ is the characteristic function of the variable $X, a>0$.

Applying this bound to the random variable whose distribution corresponds to the generating function $\rho^{\prime}(s) / \rho^{\prime}(1)$, we obtain

$$
\sup _{l} a_{l}\left[\rho^{\prime}(s) / \rho^{\prime}(1)\right] \leq(96 / 95)^{2} a^{-1} \int_{-a}^{a}\left|\rho^{\prime}\left(e^{i t}\right) / \rho^{\prime}(1)\right| d t .
$$

It is not difficult to see that

$$
a_{l}[\rho(s)]=\frac{1}{l} a_{l}\left[\rho^{\prime}(s)\right]
$$

for any $l \geq 1$; the two last relations prove the lemma.

Lemma 8. There exists a constant $M_{1}$ such that

$$
\left|G_{k}^{\prime}(s)\right| \leq M_{1} A(k) \exp \left\{-\sum_{i=1}^{k} \frac{B_{i}}{A_{i}} \Re\left(q_{i-1}(s)\right)\right\} .
$$

Proof. From the definition of $G_{k}(s)$ it follows that

$$
G_{k}^{\prime}(s)=\prod_{i=1}^{k} g_{i}^{\prime}\left(G_{i-1}(s)\right)=\exp \left\{\sum_{i=1}^{k} \ln g_{i}^{\prime}\left(G_{i-1}(s)\right)\right\} .
$$

Now, by virtue of the equalities

$$
\begin{aligned}
g_{i}^{\prime}(s) & =g_{i}^{\prime}(1)+B_{i}(s-1)+O\left((s-1)^{2}\right), \\
\ln g_{i}^{\prime}(s) & =\ln g_{i}^{\prime}(1)+\ln \left(1+B_{i}(s-1) / g_{i}^{\prime}(1)+O\left((s-1)^{2}\right)\right) \\
& =\ln g_{i}^{\prime}(1)+\frac{B_{i}}{A_{i}}(s-1)+O\left((s-1)^{2}\right)
\end{aligned}
$$

we obtain

$$
G_{k}^{\prime}(s)=A(k) \exp \left\{-\sum_{i=1}^{k} \frac{B_{i}}{A_{i}} q_{i-1}(s)+O\left(\sum_{i=1} q_{i-1}^{2}(s)\right)\right\} .
$$

Making use of inequality (37), we see that

$$
\sum_{i=1}^{k}\left|q_{i-1}(s)\right|^{2} \leq 4 \sum_{i=0}^{k-1} Q_{i}^{2}+4 \sum_{j=0}^{k-1} y_{j}^{2} .
$$

Boundedness of the former sum in the right-hand side of (46) follows from the fact that $Q_{i}=O(1 / i)$, while boundedness of the latter sum, from (23). Hence there exists a constant $M_{1}$ such that

$$
\left|G_{k}^{\prime}(s)\right| \leq M_{1} A(k)\left|\exp \left\{-\sum_{i=1}^{k} \frac{B_{i}}{A_{i}} q_{i-1}(s)\right\}\right| .
$$

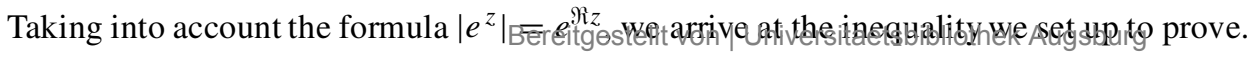


Lemma 9. There exists a positive integer $N$ such that the inequality

$$
\sum_{j=1}^{k} \frac{B_{j}}{A_{j}} \Re\left(q_{j-1}\left(e^{i t}\right)\right) \geq \frac{3}{4} \ln \left(1+\frac{(2 T(k)+1)^{2}(1-\cos t)^{2}}{\sin ^{2} t}\right)-a(N)
$$

is true for all $k>N$, where $a(N)$ is some constant.

Proof. By virtue of Lemma 6 , as $j \rightarrow \infty$,

$$
q_{j}(s)\left(\frac{(1-s)^{-1}+T(j)}{A(j)}\right) \rightarrow 1
$$

uniformly in $s$ inside the unit disk. This means that there exists $N$ such that for all $j \geq N$

$$
\Re\left(q_{j}(s)\right) \geq \frac{3}{4} \Re\left[\frac{A(j)}{(1-s)^{-1}+T(j)}\right]-\frac{1}{4}\left|\mathfrak{s}\left[\frac{A(j)}{(1-s)^{-1}+T(j)}\right]\right| .
$$

We set $s=e^{i t}$. Making use of the relation

$$
\frac{1}{1-s}=\frac{1}{(1-\cos t)-i \sin t}=\frac{1}{2}+\frac{i \sin t}{2(1-\cos t)},
$$

we obtain

$$
\begin{aligned}
& \Re\left[\frac{A(j)}{(1-s)^{-1}+T(j)}\right]=\frac{2 A(j)(2 T(j)+1)}{(2 T(j)+1)^{2}+\sin ^{2} t(1-\cos t)^{-2}}, \\
& \Im\left[\frac{A(j)}{(1-s)^{-1}+T(j)}\right]=-\frac{2 A(j)(1-\cos t) \sin ^{-1} t}{1+(2 T(j)+1)^{2}(1-\cos t)^{2} \sin ^{-2} t} .
\end{aligned}
$$

Therefore,

$$
\begin{aligned}
\sum_{j=N}^{k-1} \frac{B_{j+1}}{A_{j+1}} \Re\left(q_{j}(s)\right) \geq \frac{3}{4} \sum_{j=N}^{k-1} \frac{B_{j+1}}{A_{j+1}} \frac{2 A(j)(2 T(j)+1)}{(2 T(j)+1)^{2}+\sin ^{2} t(1-\cos t)^{-2}} \\
-\frac{1}{4} \sum_{j=0}^{k-1} \frac{B_{j+1}}{A_{j+1}} \frac{2 A(j)(1-\cos t)\left|\sin ^{-1} t\right|}{1+(2 T(j)+1)^{2}(1-\cos t)^{2} \sin ^{-2} t} .
\end{aligned}
$$

By the definition of $T(j)$,

$$
\frac{B_{j+1}}{2 A_{j+1}} A(j)=T(j+1)-T(j)=\Delta T(j) .
$$

Hence,

$$
\begin{array}{r}
\sum_{j=N}^{k-1} \frac{4(2 T(j)+1) \Delta T(j)}{(2 T(j)+1)^{2}+\sin ^{2} t(1-\cos t)^{-2}} \geq \int_{2 T(N)+1}^{2 T(k)+1} \frac{2 x d x}{x^{2}+\sin ^{2} t(1-\cos t)^{-2}} \\
=\ln \left(1+\frac{(2 T(k)+1)^{2}(1-\cos t)^{2}}{\sin ^{2} t \quad \text { Bereitgestell van | Universitaetsbin } \sin ^{2} t \text { thek Augsburg }} \begin{array}{r}
\text { Angemeldet } \\
\text { Heruntergeladen am | 18.11.19 12:40 }
\end{array}\right.
\end{array}
$$


Similarly we find that

$$
\frac{1}{4} \sum_{j=0}^{k-1} \frac{B_{j+1}}{A_{j+1}} \frac{2 A(j)(1-\cos t)\left|\sin ^{-1} t\right|}{1+(2 T(j)+1)^{2}(1-\cos t)^{2} \sin ^{-2} t} \leq \int_{0}^{\infty} \frac{d x}{1+x^{2}}=\frac{\pi}{2}
$$

Now the validity of the lemma immediately follows from (48), (49), and (50).

Lemma 10. There exists a constant $M_{2}$ such that,

$$
\left|a_{l}\left[q_{k}(s)\right]\right| \leq M_{2} \frac{A(k)}{l T(k)}
$$

for all $k, l \geq 1$.

Proof. It is obvious that $a_{l}\left[q_{k}(s)\right]=-a_{l}\left[G_{k}(s)\right]$. Therefore, it suffices to prove that (51) is true for the coefficients of the function $G_{k}(s)$.

From Lemmas 8 and 9 it follows that

$$
\left|G_{k}^{\prime}\left(e^{i t}\right)\right| \leq c_{1} A(k) T^{-3 / 2}(k) \frac{|\sin t|^{3 / 2}}{(1-\cos t)^{3 / 2}},
$$

where $c_{1}$ is some constant.

Setting $\rho=G_{k}, a=\pi / 2$ in inequality (43), we obtain

$$
a_{l}\left[G_{k}(s)\right] \leq \frac{1}{l}\left(\int_{1 / T(k)<|t|<\pi / 2}\left|G_{k}^{\prime}\left(e^{i t}\right)\right| d t+\int_{|t|<1 / T(k)}\left|G_{k}^{\prime}\left(e^{i t}\right)\right| d t\right) .
$$

The obvious bound $\left|G_{k}^{\prime}\left(e^{i t}\right)\right| \leq G_{k}^{\prime}(1)=A(k)$ implies the inequality

$$
\int_{|t|<1 / T(k)}\left|G_{k}^{\prime}\left(e^{i t}\right)\right| d t \leq 2 \frac{A(k)}{T(k)} \text {. }
$$

It is not difficult to see that

$$
\frac{|\sin t|}{|1-\cos t|} \leq \frac{2}{|t|}
$$

Applying this bound to the right-hand side of (52), we conclude that

$$
\left|G_{k}^{\prime}\left(e^{i t}\right)\right| \leq 4 c_{1} A(k) T^{-3 / 2}(k)|t|^{-3 / 2} .
$$

Therefore,

$$
\int_{1 / T(k)<|t|<\pi / 2}\left|G_{k}^{\prime}\left(e^{i t}\right)\right| d t \leq 4 c_{1} A(k) T^{-3 / 2}(k) \int_{1 / T(k)<|t|<\pi / 2}|t|^{-3 / 2} d t \leq c_{2} \frac{A(k)}{T(k)} .
$$

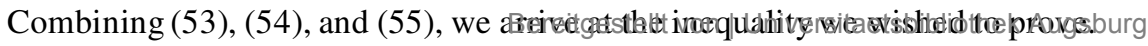


Let $\left\{\tau_{k}\right\}_{1}^{\infty}$ be a sequence of positive numbers satisfying the condition

$$
K_{\tau}=\sup _{n \geq 1} \sup _{k \geq n / 2}\left(\frac{\tau_{k}}{\tau_{n}}\right)<\infty .
$$

For any $\rho(s)$ such that $\|\rho(s)\|_{1}<\infty$ we set

$$
P_{\tau}(\rho)=\sup _{n \geq 1}\left|\rho_{n}\right| / \tau_{n}
$$

In [9], the inequality

$$
P_{\tau}\left(\rho_{1} \rho_{2}\right) \leq K_{\tau}\left(P_{\tau}\left(\rho_{1}\right)\left\|\rho_{2}\right\|_{1}+P_{\tau}\left(\rho_{2}\right)\left\|\rho_{1}\right\|_{1}\right)
$$

was established. With the use of this bound, we arrive at the following assertion.

Lemma 11. Let

$$
\lambda(t)=\sum_{i=0}^{\infty} \lambda_{i} t^{i}
$$

be a series with non-negative coefficients which converges for $|t|<t_{0}$. Then

$$
P_{\tau}(\lambda(\rho)) \leq \lambda^{\prime}\left(K_{\tau}\|\rho\|_{1}\right) P_{\tau}(\rho)
$$

for any $\rho(s)$ such that $K_{\tau}\|\rho\|_{1}<t_{0}$.

Proof. With the use of induction, from inequality (56) we derive the bound

$$
P_{\tau}\left(\rho^{i}\right) \leq H_{i}\|\rho\|_{1}^{i-1} P_{\tau}(\rho),
$$

where $H_{i}$ are defined as follows:

$$
H_{1}=1, \quad H_{i+1}=K_{\tau}\left(1+H_{i}\right) .
$$

It is clear that $K_{\tau} \geq 1$. Hence,

$$
H_{i+1} \leq K_{\tau}^{i}+K_{\tau} H_{i} \leq \ldots \leq(i+1) K_{\tau}^{i}
$$

Therefore,

$$
P_{\tau}\left(\rho^{i}\right) \leq i K_{\tau}^{i-1}\|\rho\|_{1}^{i-1} P_{\tau}(\rho)
$$

On the other hand,

$$
P_{\tau}(\lambda(\rho)) \leq \sum_{i=1}^{\infty} \lambda_{i} P_{\tau}\left(\rho^{i}\right) .
$$

Combining the two last inequalities proves the lemma.

In what follows we set $\tau_{n}=n^{-1}$ Bdteisgeketart that $K_{n}$ n 2 itaetsbibliothek Augsburg 
Lemma 12. There exists a constant $M_{3}$ such that the inequality

$$
P_{\tau}\left(C_{i}\right) \leq M_{3} \frac{A(i)}{T(i)}
$$

is true for all $i$ no less than a certain $N$.

Proof. Let $\delta(s)$ be defined as in Lemma 3. We assume that the generating function $\rho(s)$ that determines $\delta(s)$ converges in the disk of radius $R=1+t_{0}$. It is easy to verify that

$$
\delta(s)=\frac{b}{2 a^{2}} \sum_{i=2}^{\infty} \frac{\rho^{(i)}(1)}{i !}(s-1)^{i-2} .
$$

Let $q(s)$ be a power series of finite norm which satisfies the condition $\|q\|_{1}<t_{0} / K_{\tau}$. Then, by virtue of Lemma 11 ,

$$
\begin{aligned}
P_{\tau}(\delta(1-q)) & \leq P_{\tau}(q) \frac{b}{2 a^{2}} \sum_{i=3}^{\infty} \frac{\rho^{(i)}(1)}{i !}(i-2)\left(K_{\tau}\|q\|_{1}\right)^{i-3} \\
& \leq P_{\tau}(q) \frac{b}{2 a^{2}} \rho^{(3)}\left(1+K_{\tau}\|q\|_{1}\right) .
\end{aligned}
$$

From Lemma 10 it follows that

$$
P_{\tau}\left(q_{i}\right) \leq M_{2} \frac{A(i)}{T(i)} .
$$

Since $\left\|q_{i}\right\|_{1}$ and $y_{0}$ tend to zero, the inequality

$$
\left(1+2\left\|q_{i}\right\|_{1}\right)\left(1+y_{0}\right)<\frac{R+1}{2}
$$

holds for all $i \geq N$. It is easy to see that

$$
g_{i+1}^{(3)}(1+x) \leq\left(1+y_{0}\right)^{2} f^{(3)}\left((1+x)\left(1+y_{0}\right)\right) .
$$

Setting $\rho=g_{i+1}, q=q_{i}$ in (59) and taking into account the two last bounds, we obtain

$$
P_{\tau}\left(\delta_{i+1}\left(1-q_{i}\right)\right) \leq P_{\tau}\left(q_{i}\right) \frac{B_{i+1}}{2 A_{i+1}^{2}}\left(1+y_{0}\right)^{2} f^{(3)}((R+1) / 2) .
$$

Since

$$
B_{i+1}=B\left(1+O\left(y_{0}\right)\right), \quad A_{i+1}=1+O\left(y_{0}\right)
$$

and inequality (60) is true, there exists a constant $c_{1}$ such that

$$
P_{\tau}\left(\delta_{i+1}\left(1-q_{i}\right)\right) \leq c_{1} \frac{A(i)}{T(i)}
$$

The same reasoning yields

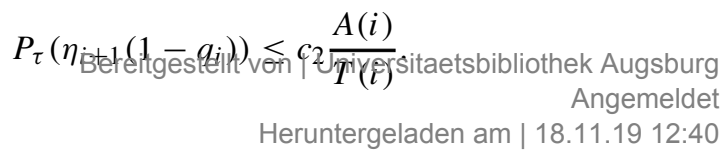


From the two last relations it follows that

$$
P_{\tau}\left(d_{i+1}\right) \leq A_{i+1} P_{\tau}\left(\delta_{i+1}\left(1-q_{i}\right)\right)+A_{i+1} P_{\tau}\left(\eta_{i+1}\left(1-q_{i}\right)\right) \leq c_{3} \frac{A(i)}{T(i)} .
$$

Further, setting $\rho=g_{i+1}, s=q_{i}$ in (34), we obtain

$$
\frac{q_{i}(s)}{q_{i+1}(s)}=\frac{1}{A_{i+1}}+\frac{2 A_{i+1}}{B_{i+1}} \delta_{i+1}\left(1-q_{i}(s)\right) \frac{q_{i}^{2}(s)}{q_{i+1}(s)} .
$$

Therefore,

$$
\frac{q_{i}(s)}{q_{i+1}(s)}=\frac{1}{A_{i+1}} \sum_{l=0}^{\infty} \frac{2^{l} A_{i+1}^{l}}{B_{i+1}^{l}} \delta_{i+1}^{l}\left(1-q_{i}(s)\right) q_{i}^{l}(s) .
$$

Applying inequality (56) to the functions $\delta_{i+1}$ and $q_{i}(s)$ and taking (61) into account, we arrive at the bound

$$
P_{\tau}\left(\delta_{i+1} q_{i}\right) \leq c_{4} \frac{A(i)}{T(i)}
$$

From this bound and Lemma 11 we find that

$$
P_{\tau}\left(q_{i} / q_{i+1}\right) \leq c_{5} \frac{A(i)}{T(i)}\left(1-\frac{2 A_{i+1}}{B_{i+1}}\left\|\delta_{i+1}\right\|_{1}\left\|q_{i}\right\|_{1}\right)^{-2} \leq c_{6} \frac{A(i)}{T(i)} .
$$

Applying inequality (56) to the functions $d_{i+1}$ and $q_{i} / q_{i+1}$, with the use of relations (62) and (63) we complete the proof of the lemma.

\section{PROOF OF THEOREM 1}

Without loss of generality we assume that $k>B n / 2$. Let

$$
y_{0}=\frac{4 k}{B^{2} n^{2}}-\frac{2}{B n} .
$$

From the hypotheses of the theorem it follows that $y_{0} \rightarrow 0$ as $n \rightarrow \infty$. It is not difficult to verify that

$$
\begin{aligned}
\mathbf{P}\left(Z_{n}=k\right) & =a_{k}\left[f_{n}(s)\right]=r_{0}^{-k} f_{n}\left(r_{0}\right) a_{k}\left[f_{n}\left(r_{0} s\right) / f_{n}\left(r_{0}\right)\right] \\
& =r_{0}^{-k}\left(1+y_{0}\right) a_{k}\left[G_{n}(s)\right]=-r_{0}^{-k}\left(1+y_{0}\right) a_{k}\left[q_{n}(s)\right] .
\end{aligned}
$$

Setting $y_{0}=4 k / B^{2} n^{2}-2 / B n$ in Lemma 1 , we obtain

$$
y_{n}=\frac{2}{B n}-\frac{1}{k}+\frac{B}{2} \gamma\left(\frac{2}{B n}-\frac{1}{k}\right)^{2} \ln (2 k / B n)+O\left(n^{-2}\right) .
$$

Removing the brackets, with the use of the inequality $\ln x<x$, we arrive at the relation

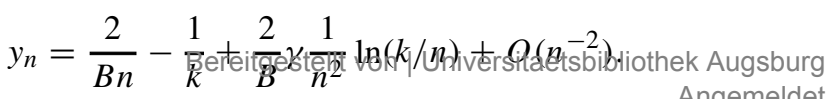


Therefore,

$$
r_{0}^{-k}=\left(1+y_{n}\right)^{-k}=\exp \left\{-\frac{2 k}{B n}+1-\frac{2}{B} \gamma \frac{k}{n^{2}} \ln (k / n)\right\}\left(1+O\left(k / n^{2}\right)\right) .
$$

Let us turn to the study of the asymptotic behaviour of $a_{l}\left[q_{n}(s)\right]$.

Lemma 5 yields

$$
q_{n}(s)=\frac{A(n)}{(1-s)^{-1}+T(n)}+\frac{q_{n}(s)}{(1-s)^{-1}+T(n)}\left(R_{N}(s)+\sum_{i=N}^{n-1} A(i) C_{i}(s) q_{i}(s)\right) .
$$

Hence,

$$
\begin{aligned}
& a_{l}\left[q_{n}(s)\right]=a_{l}\left[\frac{A(n)}{(1-s)^{-1}+T(n)}\right] \\
& \quad+a_{l}\left[\frac{q_{n}(s)}{(1-s)^{-1}+T(n)}\left(R_{N}(s)+\sum_{i=N}^{n-1} A(i) C_{i}(s) q_{i}(s)\right)\right] .
\end{aligned}
$$

It is easy to check that

$$
\frac{1}{(1-s)^{-1}+T(n)}=\frac{1}{1+T(n)}-\frac{1}{(1+T(n))^{2}} \sum_{j=1}^{\infty}\left(\frac{T(n)}{1+T(n)}\right)^{j-1} s^{j} .
$$

Therefore,

$$
\mid a_{l}\left[\frac{q_{n}(s)}{(1-s)^{-1}+T(n)}\left(R_{N}(s)+\sum_{i=N}^{n-1} A(i) C_{i}(s) q_{i}(s)\right)\right] \leq F_{1}+F_{2},
$$

where

$$
\begin{aligned}
& F_{1}=\frac{1}{1+T(n)} \mid a_{l}\left[q_{n}(s)\left(R_{N}(s)+\sum_{i=N}^{n-1} A(i) C_{i}(s) q_{i}(s)\right)\right], \\
& F_{2}=\frac{\left\|q_{n}(s)\right\|_{1}}{(1+T(n))^{2}}\left(\left\|R_{N}(s)\right\|_{1}+\sum_{i=N}^{n-1} A(i)\left\|C_{i}(s)\right\|_{1}\left\|q_{i}(s)\right\|_{1}\right) .
\end{aligned}
$$

We begin with estimating $F_{1}$. Lemmas 10,12 , and inequality (56) imply the bound

$$
P_{\tau}\left(C_{i} q_{i}\right) \leq c_{1} \frac{A(i)}{T(i)}
$$

Again making use of Lemma 10 and (56), we obtain

$$
\begin{aligned}
& P_{\tau}\left(q_{n} C_{i} q_{i}\right) \leq c_{2}\left(\left\|q_{i}\right\|_{1} \frac{A(n)}{T(n)}+\left\|q_{n}\right\|_{1} \frac{A(i)}{T(i)}\right)
\end{aligned}
$$

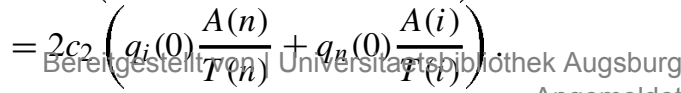


Lemma 6 guarantees the existence of constants $c_{3}, c_{4}$ such that

$$
c_{3} \frac{A(i)}{T(i)} \leq q_{i}(0) \leq c_{4} \frac{A(i)}{T(i)}
$$

for all $i$. From the two last relations we find that

$$
P_{\tau}\left(q_{n} \sum_{i=N}^{n-1} A(i) C_{i} q_{i}\right) \leq \sum_{i=N}^{n-1} A(i) P_{\tau}\left(q_{n} C_{i} q_{i}\right) \leq c_{5} \frac{A(n)}{T(n)} \sum_{i=N}^{n-1} A(i) q_{i}(0) .
$$

Lemma 3 and relation (7) imply the inequality

$$
q_{i}(0) \leq c_{6}\left(y_{n-i}+i^{-1}\right) \leq c_{6}\left(y_{0}+i^{-1}\right) .
$$

Since $A(i)$ increases,

$$
\begin{aligned}
\sum_{i=N}^{n-1} A(i) q_{i}(0) & \leq c_{6} y_{0} T(n)+c_{6} \sum_{i=1}^{n-1} A(i) i^{-1} \\
& \leq c_{6} y_{0} T(n)+c_{6} A([n / 2]) \ln n+2 c_{6} T(n) n^{-1} \leq c_{7}\left(y_{0} T(n)+\ln n\right),
\end{aligned}
$$

because $A([n / 2])$ is bounded and $n^{-1} \leq c_{8} y_{0}$. We thus obtain

$$
P_{\tau}\left(q_{n} \sum_{i=N}^{n-1} A(i) C_{i} q_{i}\right)=O\left(A(n)\left(y_{0}+T^{-1}(n) \ln n\right)\right) .
$$

Therefore, if $l \geq \alpha T(n)$, then

$$
\frac{1}{1+T(n)}\left|a_{l}\left[q_{n}(s) \sum_{i=N}^{n-1} A(i) C_{i}(s) q_{i}(s)\right]\right|=O\left(\frac{A(n)}{T^{2}(n)}\left(y_{0}+T^{-1}(n) \ln n\right)\right)
$$

for any $\alpha>0$.

By virtue of Lemmas 5 and 10,

$$
\begin{aligned}
a_{l}\left[q_{n}(s) R_{N}(s)\right] & \leq\left\|R_{N}(s)\right\|_{1} \sup _{t \geq l / 2} a_{t}\left[q_{n}(s)\right]+\left\|q_{n}(s)\right\|_{1} \sup _{t \geq l / 2} a_{t}\left[R_{N}(s)\right] \\
& \leq R \frac{2 A(n)}{l T(n)}+c_{15} \frac{A(n)}{T(n)} \sup _{t \geq l / 2} a_{t}\left[R_{N}(s)\right] .
\end{aligned}
$$

By the definition of $R_{N}(s)$,

$$
a_{l}\left[R_{N}(s)\right]=A(N) a_{l}\left[\frac{1}{1-G_{N}(s)}-\frac{1}{A(N)(1-s)}\right] .
$$

The fact that $G_{N}^{\prime \prime}(1)<\infty$ and the result due to Gelfond $[10,11]$ yield 
for $l \geq \alpha T(n)$. Therefore,

$$
\frac{1}{1+T(n)} a_{l}\left[q_{n}(s) R_{N}(s)\right]=O\left(\frac{A(n)}{T^{3}(n)}\right)
$$

for $l \geq \alpha T(n)$.

From (70) and (71) it follows that

$$
F_{1}=O\left(\frac{A(n)}{T^{2}(n)}\left(y_{0}+T^{-1}(n) \ln n\right)\right)
$$

if $l \geq \alpha T(n)$ for some $\alpha>0$.

Let us estimate $F_{2}$. By virtue of Lemmas 3, 5, and relation (69),

$$
\sum_{k=N}^{n-1} A(i)\left\|C_{i}(s)\right\|_{1}\left\|q_{i}(s)\right\|_{1} \leq 2 C \sum_{k=N}^{n-1} A(i) q_{i}(0)=O\left(T(n)\left(y_{0}+T^{-1}(n) \ln n\right)\right) .
$$

In view of the equality $\left\|q_{n}(s)\right\|_{1}=2 q_{n}(0)$ and (68),

$$
\left\|q_{n}(s)\right\|_{1}\left(\left\|R_{N}(s)\right\|_{1}+\sum_{k=N}^{n-1} A(i)\left\|C_{i}(s)\right\|_{1}\left\|q_{i}(s)\right\|_{1}\right)=O\left(A(n)\left(y_{0}+\ln n T^{-1}(n)\right)\right),
$$

which yields

$$
F_{2}=O\left(\frac{A(n)}{T^{2}(n)}\left(y_{0}+T^{-1}(n) \ln n\right)\right) .
$$

From (66), (72), (67), and (73) it follows that the relation

$$
a_{l}\left[q_{n}(s)\right]=-\frac{A(n)}{T^{2}(n)}\left(\frac{T(n)}{1+T(n)}\right)^{l-1}+O\left(\frac{A(n)}{T^{2}(n)}\left(y_{0}+T^{-1}(n) \ln n\right)\right)
$$

is true for all $l \geq \alpha T(n)$. Hence,

$$
a_{l}\left[q_{n}(s)\right]=-\frac{A(n)}{T^{2}(n)} \exp \left\{-\frac{l}{T(n)}\right\}\left(1+O\left(y_{0}+T^{-1}(n) \ln n\right)\right)
$$

holds uniformly in $\alpha T(n) \leq l \leq \beta T(n)$, where $\alpha, \beta, \alpha<\beta$, are arbitrary real numbers.

From the definition of $y_{0}$ and formulas (27), (28) it follows that

$$
T(n)=k\left(1+O\left(y_{0}\right)\right), \quad \frac{A(n)}{T^{2}(n)}=\frac{4}{B^{2} n^{2}}\left(1+O\left(y_{0}\right)\right) .
$$

Setting $l=k$ in (74), we obtain

$$
a_{k}\left[q_{n}(s)\right]=-\frac{4}{B^{2} n^{2}} e^{-1}\left(1+O\left(k n^{-2}+k^{-1} \ln n\right)\right) .
$$

This equality and formulas (64), (65)Bpreivgethel theoremiversitaetsbibliothek Augsburg 


\section{PROOF OF THEOREM 2}

First we assume that $k \geq \lambda n \ln n$, where $\lambda$ is chosen so that the relation

$$
r_{0}^{-\lambda n \ln n}=O\left(n^{-1}\right)
$$

holds for any $k \geq \lambda n \ln n$. The existence of such $\lambda$ follows from (65).

Furthermore, by virtue of Theorem 1

$$
\mathbf{P}\left(Z_{n} \geq k\right)=\sum_{i=k}^{2 k-1} \frac{4}{B^{2} n^{2}}\left(\exp \left\{-\frac{2 i}{B n}-\frac{2 \gamma i}{B n^{2}} \ln \left(\frac{i}{n}\right)\right\}\right)\left(1+O\left(k n^{-2}\right)\right)+\mathbf{P}\left(Z_{n} \geq 2 k\right)
$$

Let $\gamma>0$. Then

$$
\begin{aligned}
\sum_{i=k}^{2 k-1} \exp \left\{-\frac{2 i}{B n}-\frac{2}{B} \gamma \frac{i}{n^{2}} \ln \left(\frac{i}{n}\right)\right\} & \leq \exp \left\{-\frac{2 k}{B n}-\frac{2}{B} \gamma \frac{k}{n^{2}} \ln \left(\frac{k}{n}\right)\right\} \sum_{i=0}^{\infty} e^{-2 i / B n} \\
& =\frac{B n}{2} \exp \left\{-\frac{2 k}{B n}-\frac{2}{B} \gamma \frac{k}{n^{2}} \ln \left(\frac{k}{n}\right)\right\}\left(1+O\left(n^{-1}\right)\right) .
\end{aligned}
$$

On the other hand, in view of the constraints imposed on $\lambda$,

$$
\begin{aligned}
& \sum_{i=k}^{2 k-1} \exp \left\{-\frac{2 i}{B n}-\frac{2}{B} \gamma \frac{i}{n^{2}} \ln \left(\frac{i}{n}\right)\right\} \\
& \geq \exp \left\{-\frac{2 k}{B n}-\frac{2}{B} \gamma \frac{k}{n^{2}} \ln \left(\frac{2 k}{n}\right)\right\} \sum_{i=0}^{k-1} \exp \left\{-\frac{2 i}{B n}-\frac{2}{B} \gamma \frac{i}{n^{2}} \ln \left(\frac{2 k}{n}\right)\right\} \\
& \quad=\frac{B n}{2} \exp \left\{-\frac{2 k}{B n}-\frac{2}{B} \gamma \frac{k}{n^{2}} \ln \left(\frac{k}{n}\right)\right\}\left(1+O\left(k n^{-2}\right)\right) .
\end{aligned}
$$

From the two last relations we find that

$$
\begin{aligned}
\sum_{i=k}^{2 k-1} \exp \left\{-\frac{2 i}{B n}-\frac{2}{B} \gamma \frac{i}{n^{2}}\right. & \left.\ln \left(\frac{i}{n}\right)\right\} \\
& =\frac{B n}{2} \exp \left\{-\frac{2 k}{B n}-\frac{2}{B} \gamma \frac{k}{n^{2}} \ln \left(\frac{k}{n}\right)\right\}\left(1+O\left(k n^{-2}\right)\right) .
\end{aligned}
$$

for $\gamma>0$. Similar reasoning proves the validity of (78) for $\gamma<0$ as well.

Let us estimate the second term in the right-hand side of (77). It is not difficult to see that

$$
\mathbf{P}\left(Z_{n} \geq 2 k\right) \leq s^{-2 k}\left(f_{n}(s)-f_{n}(0)\right)
$$

for any $s \geq 1$. Setting $s=r_{0}$ in this inequality, we arrive at

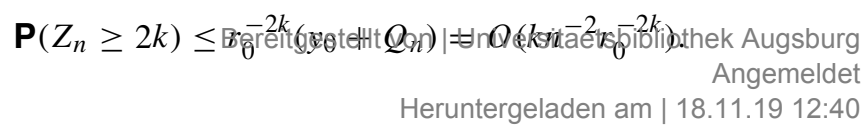


In view of (65) and the choice of $\lambda$,

$$
\mathbf{P}\left(Z_{n} \geq 2 k\right)=O\left(k n^{-3} r_{0}^{-k}\right)=O\left(\frac{k}{n^{3}} \exp \left\{-\frac{2 k}{B n}-\frac{2}{B} \gamma \frac{k}{n^{2}} \ln \left(\frac{k}{n}\right)\right\}\right) .
$$

From (77), (78), and (79) we obtain (11).

It remains to demonstrate that (11) is true for $k \leq \lambda n \ln n$. From (10) we see that

$$
\mathbf{P}\left(Z_{n}=i\right)=\frac{4}{B^{2} n^{2}} \exp \left\{-\frac{2 i}{B n}-\frac{2 \gamma}{B} \frac{i}{n^{2}} \ln \left(\frac{i}{n}\right)\right\}\left(1+O\left(n^{-1} \ln n\right)\right)
$$

uniformly in $i$ such that $i=O(n \ln n)$. Therefore,

$$
\begin{array}{r}
\mathbf{P}\left(Z_{n} \geq k\right)=\left(\sum_{i=k}^{k+\lambda n \ln n} \frac{4}{B^{2} n^{2}} \exp \left\{-\frac{2 i}{B n}-\frac{2 \gamma}{B} \frac{i}{n^{2}} \ln \left(\frac{i}{n}\right)\right\}\right)\left(1+O\left(n^{-1} \ln n\right)\right) \\
+\mathbf{P}\left(Z_{n} \geq k+\lambda n \ln n\right) .
\end{array}
$$

Reasoning as in the proof of (78), we obtain

$$
\begin{aligned}
\sum_{i=k}^{k+\lambda n \ln n} \exp \left\{-\frac{2 i}{B n}-\frac{2}{B} \gamma \frac{i}{n^{2}}\right. & \left.\ln \left(\frac{i}{n}\right)\right\} \\
& =\frac{B n}{2} \exp \left\{-\frac{2 k}{B n}-\frac{2}{B} \gamma \frac{k}{n^{2}} \ln \left(\frac{k}{n}\right)\right\}\left(1+O\left(n^{-1} \ln n\right)\right)
\end{aligned}
$$

The asymptotic behaviour of the second term in the right-hand side of (80) has been cleared up while studying the case $k \geq \lambda n \ln n$. We thus arrive at the equality

$$
\begin{aligned}
\mathbf{P}\left(Z_{n}\right. & \geq k)=\frac{2}{B n} \exp \left\{-\frac{2 k}{B n}-\frac{2}{B} \gamma \frac{k}{n^{2}} \ln \left(\frac{k}{n}\right)\right\}\left(1+O\left(n^{-1} \ln n\right)\right) \\
& +\frac{2}{B n} \exp \left\{-\frac{2 k+\lambda n \ln n}{B n}-\frac{2}{B} \gamma \frac{k+\lambda n \ln n}{n^{2}} \ln \left(\frac{k+\lambda n \ln n}{n}\right)\right\}\left(1+O\left(n^{-1} \ln n\right)\right) .
\end{aligned}
$$

It is not difficult to see that the constraint imposed on $\lambda$ implies the relation

$$
\begin{aligned}
\exp \left\{-\frac{2 k+\lambda n \ln n}{B n}-\frac{2}{B} \gamma \frac{k+\lambda n \ln n}{n^{2}} \ln \left(\frac{k+\lambda n \ln n}{n}\right)\right\} \\
\quad \leq \frac{c}{n} \exp \left\{-\frac{2 k}{B n}-\frac{2}{B} \gamma \frac{k}{n^{2}} \ln \left(\frac{k}{n}\right)\right\} .
\end{aligned}
$$

Therefore,

$$
\mathbf{P}\left(Z_{n} \geq k\right)=\frac{2}{B n} \exp \left\{-\frac{2 k}{B n}-\frac{2}{B n} \gamma \frac{k}{n^{2}} \ln \left(\frac{k}{n}\right)\right\}\left(1+O\left(n^{-1} \ln n\right)\right) .
$$

Theorem 2 is thus proved.

In conclusion, we would like to express our gratitude to the reviewer who directed the authors' attention to a series of inaccuracies and misprints and made a series of remarks which served to improve the presentationitof sthetresults niversitaetsbibliothek Augsburg 


\section{REFERENCES}

1. T. E. Harris, The Theory of Branching Processes. Springer, Berlin, 1963.

2. S. V. Nagaev and R. Mukhamedkhanova, Some limit theorems of the theory of branching processes. In: Limit Theorems and Statistical Inferences. Fan, Tashkent, 1966, pp.90-112 (in Russian).

3. S. V. Nagaev and N. V. Vahrusev, Estimation of probabilities of large deviations for a critical Galton-Watson process. Theory Probab. Appl. (1975) 20, 179-180.

4. V. V. Petrov, Sums of Independent Random Variables. Akademie, Berlin, 1975.

5. G. D. Makarov, Large deviations for a critical Galton-Watson process. Theory Probab. Appl. (1981) 25, 481-492.

6. K. A. Borovkov, Analysis of transient effects for branching processes. Theory Probab. Appl. (1994) 39, 379-395.

7. H. Kesten, P. Ney, and F. Spitzer, The Galton-Watson process with mean one and finite variance. Theory Probab. Appl. (1966) 11, 513-540.

8. L. H. Loomis, An Introduction to Abstract Harmonic Analysis. Van Nostrand, Toronto, 1953.

9. B. A. Rogozin, Asymptotic behaviour of the coefficients in Levy-Wiener theorems on absolutely convergent trigonometric series. Sib. Math. J. (1973) 14, 1304-1312 (in Russian).

10. A. O. Gelfond, An estimate for the remainder term in a limit theorem for recurrent events. Theory Probab. Appl. (1964) 9, 299-303.

11. B. A. Rogozin, An estimate of the remainder term in limit theorems of renewal theory. Theory Probab. Appl. (1973) 18, 662-677. 